\title{
Character Recognition using Neural Network Self- Organizing Map (NN-SOM)
}

\author{
Okpor Margaret Dumebi \\ Department of Computer Science \\ Delta State Polytechnic, Ozoro \\ Delta State, Nigeria
}

\begin{abstract}
Understanding the neuron-logic and natural sense with which humans can recognize textual pattern and characters had actually called for computational artifact to mimic human behaviour. Character recognition is a key part of applying processing to safeguard transcribed data in order to recover it at a later stage, just as encouraging its method of correspondence utilizing computational intelligence and data mining approach. Character recognition with computing gadget allows easy access, content storage and distributed capacity. In this work, self-organizing map of the neural network was used to distinguish alphabetic characters by assigning them to different bins; using the ASCII values to represent each of the graphic characters and train the network with anticipated responses to recognize them. Each line has a $5 \times 7$ dot representation of each character with simple 3-bit representation; each of the output categories was named as well with a binary map of 35 pixel values. The simulator completed the learning in fewer cycles, and training patterns were learned very well while smaller tolerance was used. The results showed each foreign character in the match category closest to it. The middle layer of the network acts as a feature detector with much influence on training time and generalization capability.
\end{abstract}

\section{Keywords}

Neural Network, Self- Organizing Map, Input Vector, Pixel Signal, Textual Pattern

\section{INTRODUCTION}

Character recognition describes the process of using personal computer or associated digital gadgets to retrieve and clarify textual documents from different input documentations like touch-screens, photographs and many other devices. It typically addresses many algorithmic rules in machine learning, which is applicable to text, speech, computer vision, and other areas. Because of interest in revealing and understanding the mystery of how people can perceive manually written examples, endeavors are being made to copy human conduct. Character recognition is such a part of computational intelligence where "computers" are utilized to decipher and safeguard literary archive or printed data in order to recover it at a later stage just as encouraging its method of correspondence utilizing AI approach [10].

In spite of the reluctance for the tremendous progression in innovation that encourages viable composition and AI, [12] said the vast majority of people actually want to take their notes customarily with pen and paper. Notwithstanding, there are downsides to manually written content. These difficulties incorporates, however not restricted to the challenges in putting away and getting to reports in physical structure in an effective way, search through records productively and to impart them to other people. Computers can store enormous measure of data in a little chip subsequently limiting extra room; among different advantages of computer incorporates speedy looking, quicker recovery, simple adjustment, and finding new data from existing information.

The capacity of the computer to decipher an individual penmanship, and furthermore keep up the uniqueness of such composition, is known as penmanship acknowledgment [10]. Albeit different information gadgets have been created to give offices to clients, for example, console, mouse, computerized pen, pointer, and contact screen. Thusly, the presence of computer penmanship acknowledgment frameworks permits clients to effectively record and overcome any issues between the gifted computer clients and the individuals who are definitely not. [7] isolated computer penmanship into on-line penmanship acknowledgment and disconnected composing acknowledgment. The capacity of the computer to comprehend checked composing is called disconnected penmanship acknowledgment while online hand-composing alludes to the way toward perceiving penmanship through touch cushion utilizing pointer pen.

The mix of computational intelligence and data mining method signifies a machine learning algorithm that is recently applied in unraveling countless, heterogeneous, and nonreasonable datasets which are not restricted to text, sound and picture datasets [6].

There are lots of processing bottlenecks associated with document files; these challenges include the difficulties in storing and accessing physical documents in an efficient manner; searching through documents for cross-platform conversion and communication. Thus, a self-organizing model of neural network can handle feature extraction and content mapping for transforming textual documentation or transaction documents to electronic and/or digital text.

This work is aimed at providing and experimenting with a self-organizing model of neural network for character recognition. The objectives are:

i. Design a self-organizing model using neural network (NN-SOM).

ii. Experiment with the model in (i) and evaluate the performance

\section{LITERATURE REVIEW}

Character recognition is a key part of applying figuring to protect literary data to recover it at a later stage, just as encouraging its method of correspondence utilizing computational insight and information mining approach. Character recognition with registering contraption permits simple access, content stockpiling and disseminated limit. Computer is utilized to communicate compositions into computerized media; current innovation utilizes an info gadget 
to catch the penmanship of a client, which could be made utilizing "pen and paper". This caught text is changed over into advanced content arrangement by changing manually written exchange archives [4].

\subsection{Neural Networks (NN)}

An artificial neural organization is a theoretical computational model of the human mind. The human mind has an expected 1011 small units called neurons. These neurons are interconnected with an expected 1015 connections. Like the cerebrum, an ANN is made out of counterfeit neurons (or handling units) and interconnections. A neural organization, as the name shows, is an organization structure comprising of various hubs associated through directional connections. Every hub speaks to a preparing unit, and the connections between hubs determine the causal connection between associated hubs [11] All hubs are versatile, which implies that the yields of these hubs rely upon modifiable boundaries relating to these hubs [5]

\subsection{Self-Organizing Map (SOM)}

This is a neural network system developed by Teuvo Kohonen which inculcates self-organizing properties in the network, in order to infer relationships and learn more as more inputs are presented to it. One major advantage of this network scheme is the expectation for the system to change with changing conditions and inputs, as well as constant or continuous learning [1] and as such it is also known as Kohonen feature map or Kohonen SOM. It may be used by itself or as a layer of another neural network thus composed of neurons that compete with each other using a winner-take-all strategy [9].
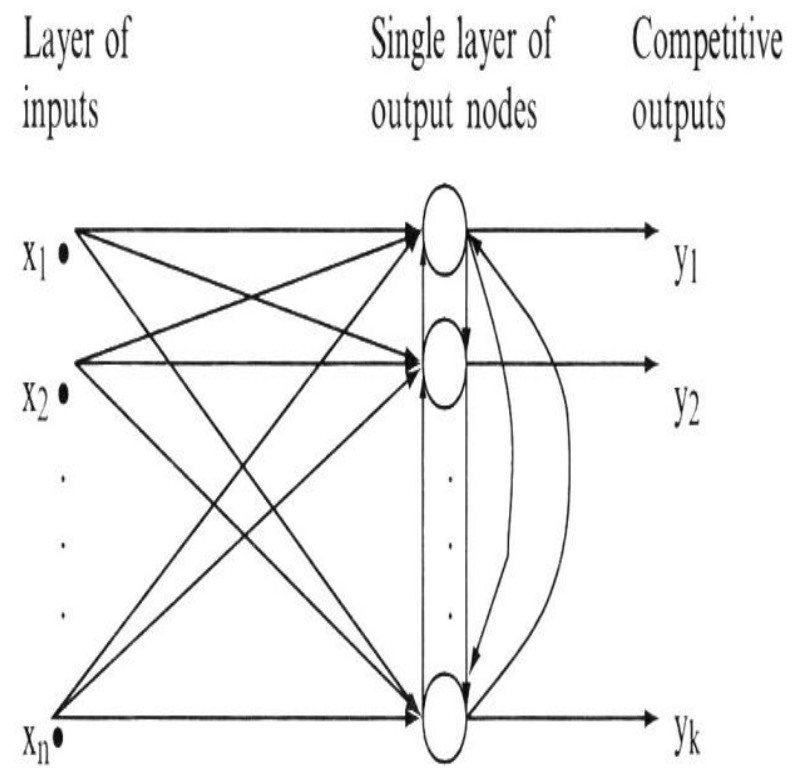

Fig. 1: Competitive Learning Architecture of Neural Network (Bhatia, 2014)

\subsection{Related Work and Appraisal}

Textual document can be changed over into advanced structure, [14]. presented how printed archive is changed over into computerized structure and bit of leeway, weakness of Digital Pen. Their work depicts how electronic or computerized pen cooperate with common paper to catch pen strokes on a small camera. It recorded different capacities of the framework to incorporate chronicle exact penmanship picture and its composed structure. They likewise demonstrated how dataset can be transferred by means of all around coordinated cell phone or a Personal (computer). Their work portrayed how advanced pen communicates composing into computerized media and specified the benefits of Digital Pen.

[13] Introduced the Identification of Personality through Handwriting Analysis. Their work gave investigation audit on conduct, and the technique used to distinguish this character through penmanship examination and the current condition ofworkmanship related to it. Through the work actualized different character investigation procedures so as to accomplish the exact data with respect to the quirk of every character. A few components have being found to have influenced the rightness of graphology. These components remember equivocalness for penmanship just as human mistake, the examination of their work explicitly discovered forecast to be around $90 \%$. Be that as it may, independence order assessment of penmanship will achieve an improved and supportive framework. Particularly, since Artificial neural organizations license a framework to be pre-prepared and distinguish attributes of penmanship and render to an adjusting character characteristic.

[7] introduced on a cursive English characters by means of an Optical Character Recognition system. In their work, they had the option to analyze fundamentally how cursive characters can be checked and how their photos can be taken care of into the computer with the end goal for it to be acknowledged utilizing a model called Hidden Markov Mode. This thus does a change into similar words identical to printed characters. In other to thoroughly evade some rising blunders they had the option to plan a calculation to deal with these difficulties. Middle channels were utilized to dodge blunders coming about because of clamor [3].

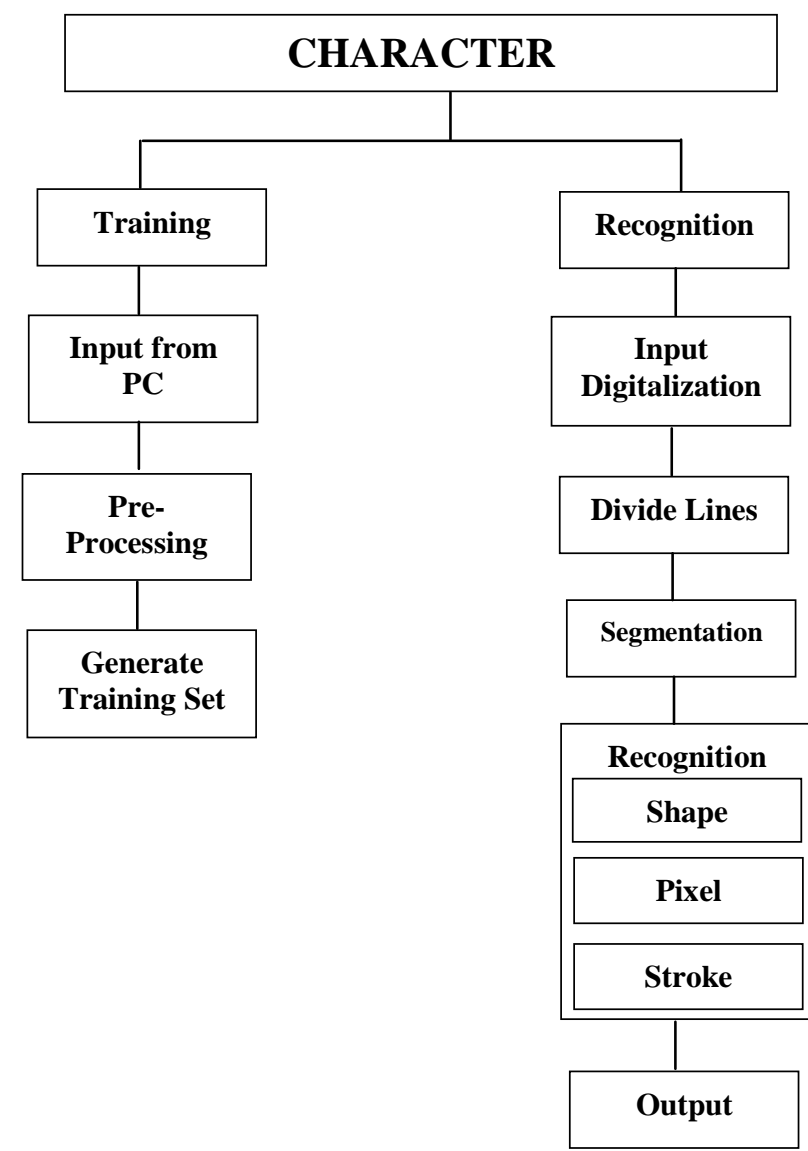

Fig 2: Generic Architecture of the Character Recognition System [11] 


\section{METHODOLOGY}

Inputs are fed into each of the neurons in the output (Kohonen) layer (from the input layer). Each neuron determines its output according to a weighted sum formula:

Output $=\Sigma \mathrm{w}_{\mathrm{ij}} \mathrm{X}_{\mathrm{i}}$.

The weights and the inputs are generally standardized, which implies that the extents of the weight and information vectors are set equivalent to one. The neuron with the biggest yield is the champ. This neuron has a last yield of 1. All different neurons in the layer have a yield of zero. Varying information designs wind up terminating diverse victor neurons. Comparable or indistinguishable information designs order to a similar yield neuron.

\subsection{Normalization of a Vector}

Consider a vector, $\mathrm{A}=a x+b y+c z$. The standardized vector $\mathrm{A}^{\prime}$ is gotten by isolating every segment of $\mathrm{A}$ by the square foundation of the aggregate of squares of the apparent multitude of parts. As such every part is multiplied by 1/ [radic] $\left(a^{2}+b^{2}+c^{2}\right)$. Both the weight vector and the input vector are standardized during the activity of oneself arranging map. The explanation behind this is the preparation law utilizes deduction of the weight vector from the input vector. Using normalization of the values in the subtraction reduces both vectors to a unit-less status

$\mathbf{W}_{\text {new }}=\mathbf{W}_{\text {old }}+$ alpha $*\left(\right.$ Input $\left.-\mathbf{W}_{\text {old }}\right)$

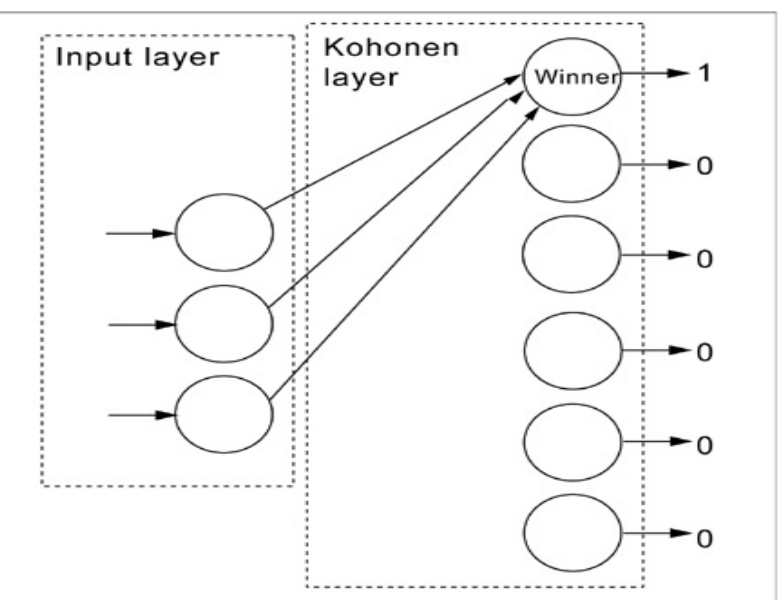

Fig. 3: Kohonen Network - Self Organizing Map (NNSOM)

\subsection{Characters' Representation}

Each character is represented by a $5 \times 7$ grid of pixels, graphical printing characters of the extended ASCII character set to show a gray scale output for each pixel. For instance, letter A was represented by the pattern shown in figure 4 and the blackened boxes represent value 1, while empty boxes represent a zero, for all characters with a binary map of 35 pixel values.

Letter A representation by binary values is:

00100

01010

100001

100001

11111

100001

100001

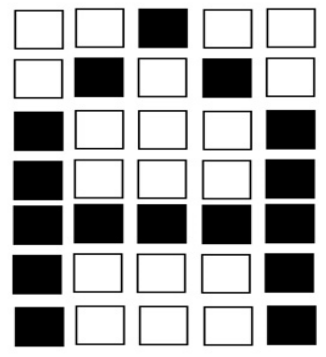

Fig 4: Letter A representation with a $5 \times 7$ pattern signal

Table 1: Quantizing the Weight Vector

\begin{tabular}{|c|c|}
\hline$<=0$ & White rectangle (space) \\
\hline \hline $0<$ weight $<=0.25$ & Light-dotted rectangle \\
\hline \hline $0.25<$ weight $<=0.50$ & Medium-dotted rectangle \\
\hline \hline $0.50<$ weight $<=0.75$ & Dark-dotted rectangle \\
\hline \hline weight $>0.75$ & Black rectangle \\
\hline
\end{tabular}

Table 2: Rectangle Graphic Characters and ASCII Values

\begin{tabular}{|l|l|}
\hline White rectangle & 255 \\
\hline Light-dotted rectangle & 176 \\
\hline \hline $\begin{array}{l}\text { Medium-dotted } \\
\text { rectangle }\end{array}$ & 177 \\
\hline Dark-dotted rectangle & 178 \\
\hline Black rectangle & 219 \\
\hline
\end{tabular}

\section{SIMULATION AND DISCUSSION}

Scientific experiment was shown in a one-dimensional Selforganizing Map, whereby Neurons in a 2-D layer learn to represent various religions of the input space where input vectors happen. Furthermore, neighboring neurons figure out how to react as the layer learns the geography of the introduced input space and to plan the example signal for each given character.

\subsection{Experimental Environment}

Simulation was performed on HP 630 laptop computer with 2.1GHz Dual-Core processor, 3.00GB RAM and 250GB Hard disk on Microsoft Windows 10 Pro. Neural network and selforganizing tools of MATLAB were used for the simulation and analysis of network structure. However, the minimum hardware and software requirements for modeling are:
i. $\quad 512 \mathrm{MHz}$ speed of system processor
ii. ii. $256 \mathrm{MB}$ RAM of the computer memory
iii. 20GB hard disk of the internal storage
iv. 15 " inch SVGA of computer monitor
v. MATLAB 6.0
vi. Windows XP 


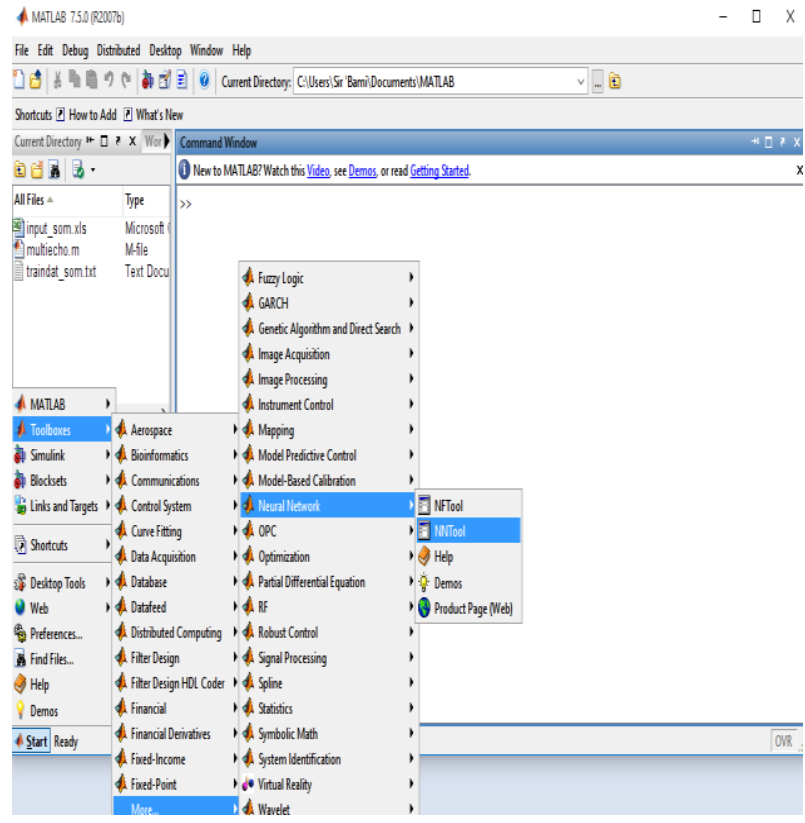

Fig. 5: Main Window of MATLAB simulation workspace

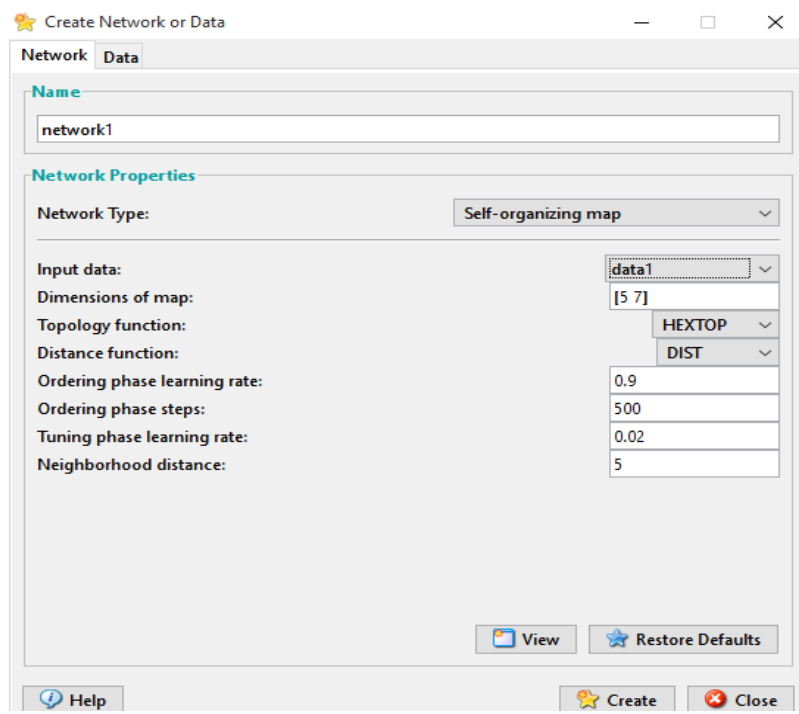

Fig 6: Neural Network - Network Manager Window in MATLAB

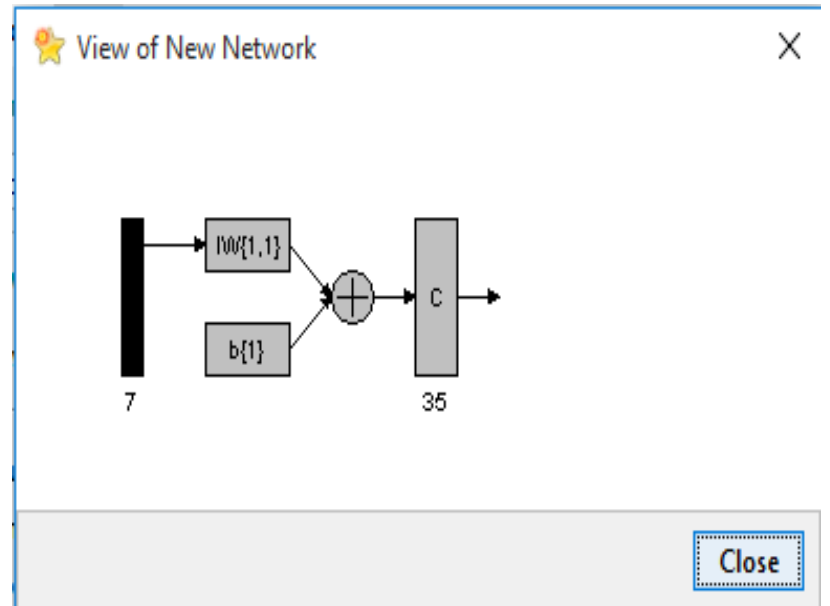

Fig 7: Neural Network - SOM Structure in MATLAB

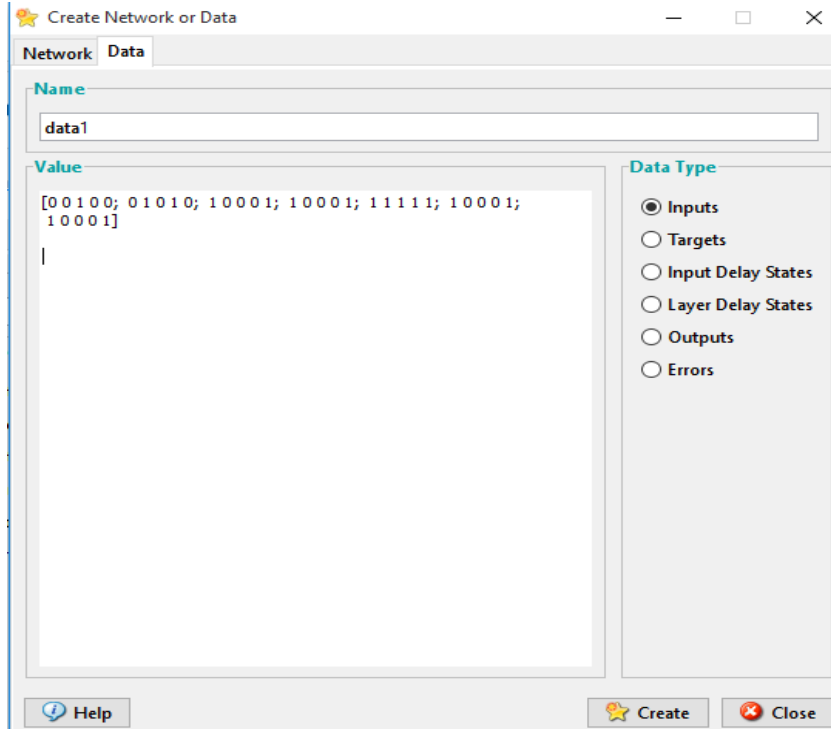

Fig 8: Neural Network - Data Manager Window in MATLAB

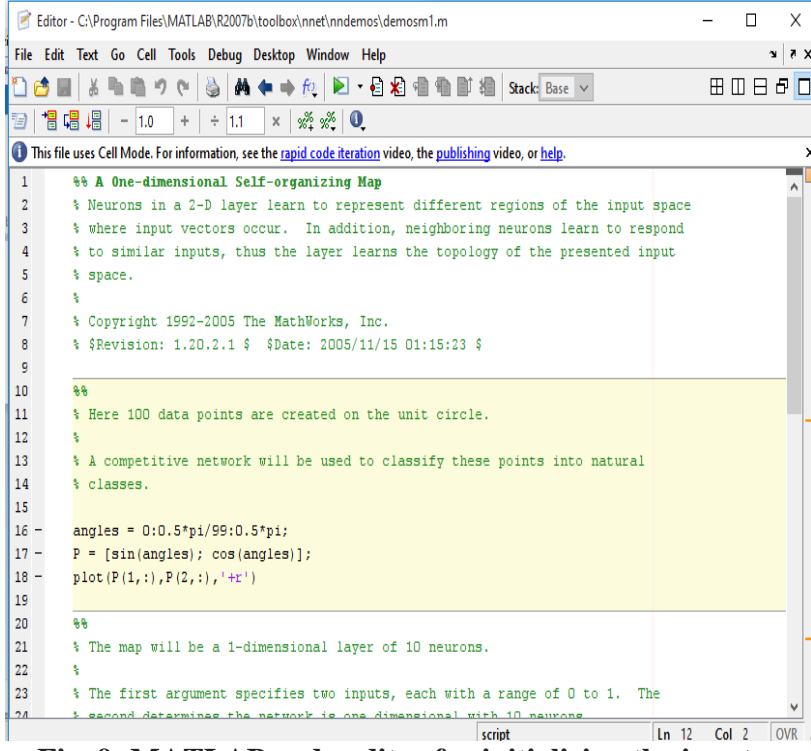

Fig. 9: MATLAB code-editor for initializing the input pattern

The first argument specifies two inputs, each with a range of 0 to 1 . The second determines the network is one dimensional with 10 neurons.

net $=$ newsom $\left(\left[\begin{array}{lll}0 & 1 ; 0 & 1\end{array}\right],[10]\right)$;

Specify the network is to be trained for 10 epochs and use TRAIN to train the network on the input data

P:net.trainParam.epochs $=10 ; \quad$ net $=\operatorname{train}($ net,$P)$;

TRAINR, Epoch 0/10

TRAINR, Epoch 10/10

TRAINR, Maximum epoch reached.

Then, the trained network was plotted with PLOTSOM. The red dots are the neuron's weight vectors, and the blue lines connect each pair within a distance of 1 .

plotsom(net.iw $\{1,1\}$,net.layers $\{1\}$.distances) 


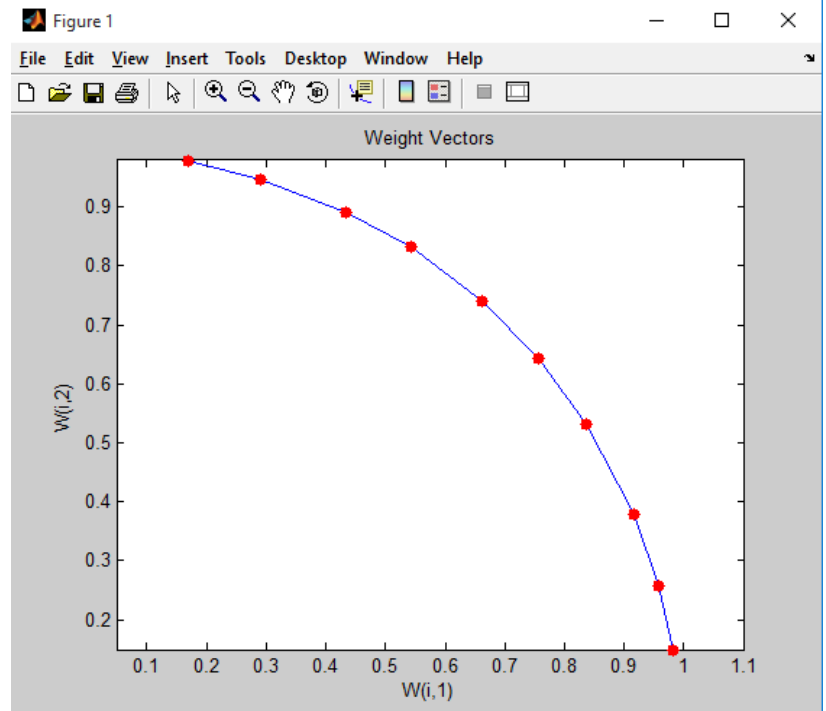

Fig. 10: Training Plot for NN-SOM in MATLAB

Hence, the map was used to classify inputs, like $[1 ; 0]$ :

Either neuron 1 or 10 ought to have a yield of 1 , as the above information vector was toward one side of the introduced input space. The main pair of numbers show the neuron, and the single number demonstrates its yield.

$$
\begin{gathered}
\quad \mathrm{p}=[1 ; 0] ; \\
\mathrm{a}=\operatorname{sim}(\text { net,p) } \\
\mathrm{a}=\quad(1,1)
\end{gathered}
$$

\section{CONCLUSION}

The character recognition model is designed to capture textual information or character-based content of users; perform feature extraction on the character sets by implementing neural alphabetic and pattern classifier with self-organizing capability. The functional logic of system model moves from the problem to the solution domain for transforming conventional transaction documents to digitized text with selforganization pattern of character and textual signals.

The simulation of character recognition model has shown to have many potential cognitive benefits such as simple and relaxed writing posture. The predominant input mechanism for data entry might not be ideal for all persons in business activities and daily transaction. For instance, people who suffer from repetitive strain injuries (RSI) and related disabilities may find typing on a keyboard difficult or even impossible.

The overall improvement on recognition engine and pattern classification had been able to address three main types of error which includes the removal of unnecessary space errors, certifying the last character of a statement eliminates errors in the system and remembers to concatenate each together.

\section{REFERENCES}

[1] Abdullah A.M. Al-Harigy M.L and. Al-Fraidi H.H. 2012. Off-line arabic handwriting character recognition using word segmentation Journal of Computing, Vol 4(3), pp $40-41$.

[2] Abdel-Hamid O. Mohamed A.R, H.L. Jiang, G. Deng, and D.Yu, 2014. Convolutional neural networks for speech recognition, IEEE Transactions on Audio, Speech and Language Processing, Vol 22(10), pp 1533-1545.

[3] Ahmed M.O., El-Bakry M.L, Eldosuky A.I. and Shehab I., 2016. Handwritten text recognition system based on natural network, International Journal of Advanced Research in Computer Science and Technology, Vol 4(1), pp 111-120.

[4] I. Almusaly, and R. Metoyer. 2015. A syntax-directed keyboard extension for writing source code on tourch screen devices. In Visual Languages and Human-Centric Computing, vol 3(34), pp 195-202.

[5] Amma, C., Marcus G. and Tanja S. 2014. Air writing: a wearable handwriting recognition system. Personal And Ubiquitous Computing. Vol 18(1), pp ,

[6] Amri A.A., Ismail R.A and.Zarir A.A. 2018 International Journal of Advanced Computer Science and its Applications, Vol 9(2), pp 32 -40.

[7] Aparna A, and Muthumani I. 2014. Optical character recognition for handwritten cursive english charcters. International Journal of Computer Science and Information Technologies, Vol 5(1), pp 847-848.

[8] Bhatia N. 2014. Optical character recognition techniques. International Journal of Advanced Research in Computer Science and Software Engineering, Vol 4(5), pp 221 226.

[9] Chakravarthy A.S.,.Raja V.P and Aavadhani P.S., 2011. Handwritten text image authentication using back propagation. International Journal of Network Security and Its Applications (IJNSA), 3(5), 121 -130.

[10] Esau G. 2018. An enhanced computer handwriting recognition using deep learning approach. M.Sc Dissertation: Rivers State University, Port Harcourt.

[11] M. Farha, G. Srinivasa, A.J.,Ashwin and H.K Hemanth, 2013. Online hand written character recognition. Journal of Computer Engineering, Vol 11(5), 30-36.

[12] Fortunati L. and Vincent J. 2012. Sociological insights on the comparison of writing and reading on paper with writing and reading digitally. Vol 2(5), pp 25-35.

[13] Kedar S., Nair V., and Kulkarni S. 2015. Personality identification through handwriting analysis: a review. International Journal of Advanced Research in Computer Science and Software Engineering, Vol 5(1), pp549-556.

[14] Saoji R.S., and Dande A.A. 2016 .Digital pen: how written document convert into digital form, International Journal of Engineering Trends and Technology, Vol 36(4), pp 180- 183. 